\title{
Network based Load-Aware Mobility Management in IEEE 802.11 Wireless Mesh Networks
}

\author{
Zheng Wang ${ }^{1,2, *}$ \\ ${ }^{1}$ Computer Network Information Center, Chinese Academy of Sciences, Beijing 100190, China \\ ${ }^{2}$ China Organizational Name Administration Center, Beijing 100028, China
}

Received: 18 Apr. 2013, Revised: 19 Aug. 2013, Accepted: 21 Aug. 2013

Published online: 1 Mar. 2014

\begin{abstract}
One major concern for mobile networks consists of finding efficient ways of handling user mobility so that the handover process has minimum effect on users' ongoing sessions. Although COAP provides an efficient way for mobility management in IEEE 802.11 wireless mesh networks, it does not control load among multiple MAPs in the network. Thus, in many cases, the attached MAP is overloaded, and extensive delays are experienced during the routing process. To tackle this problem, this paper proposes a load-aware mobility management scheme. The overloaded MAP is detected according to the load estimation. Then the overloaded MAP initiates the search procedure to find the underutilized MAP and transfer the MN's attachment request to it. The proposed scheme can efficiently balance the handover load among MAPs at the cost of slightly prolonged attachment delay and increased attachment messages compared with COAP.
\end{abstract}

Keywords: handover scheme, wireless mesh network, load-aware, mobility management

\section{Introduction}

The IEEE 802.11 wireless local area networks (WLANs) were designed to act as an infrastructure to provide wireless Internet access to homes, businesses and public spaces. The original design gives a solution to the networking problem where the stations (STAs) are wirelessly connected to the available access points (APs), and the APs are connected to a wired backbone network. As an emerging IEEE 802.11standard, 802.11s enables mesh networks where a wireless back bone network is set up in order to support end-to-end wireless user communication [1]. The mesh node can not only participate in traffic forwarding but also serve an AP, thereby becomes a Mesh Access Point (MAP).

Load balancing is an important consideration for 802.11 networks [2]. To send and receive frames, a wireless station with an 802.11 interface uses an AP to a wired infrastructure. APs essentially bridge the wireless and wired worlds, serving as link-layer attachment points to the Internet. Because wireless stations independently select APs to camp on, traffic loads might be unevenly shared, leading to overloading and network congestion. The result is low data throughput for both the system and users. Traffic load problems are most likely to occur in public access areas such as stations, airports, and convention or exhibition sites. Researchers have developed several solutions to this problem, although existing designs continue to exhibit insufficiencies.

Mobility management has been an active research area in recent years. The objective is to enable seamless handover when a Mobile Node (MN) moves from one network to another network. Neighboring 802.11APs, probably under different administrative domains, can form wireless mesh networks. Thus, it is desirable to enable transparent roaming of MN within such mesh networks. To facilitate easy deployment, it should not require modification on MN. Proxy Mobile IPv6 (PMIPv6) [3] requires no modification of network stack on the mobile nodes. However, PMIPv6 only allows MN to roam among different wireless networks under the same administrative PMIPv6 domain. To enable transparent roaming of $\mathrm{MN}$ among different administrative domains, a network based local mobility scheme based on Cooperative AP (COAP) for 802.11wireless is proposed as an alternative to PMIPv6 [4].

One important issue, which is highly missed in the design of Mobility management schemes such as

\footnotetext{
*Corresponding author e-mail: zhengwang09@126.com
} 
HMIPv6 [5] and COAP, is on the traffic load and processing power distribution over multiple MAPs in a large mobile network. Indeed, in the case where a single MAP administers a large domain, the distance between MNs and the MAP can add significant delays to packets and affect route optimization as MNs move away from the MAP. Operators of large mobile networks may, thus, choose to deploy several MAPs in one domain. In such large networks with multiple MAPs, it is easily possible that some MAPs become congested, whereas other MAPs are underutilized. To cope with such an issue, which affects the overall network performance, a load-aware mobility management strategy is required. In this paper, a load-aware mobility management scheme in IEEE 802.11 wireless mesh networks based on COAP (LA-COAP) is proposed. The overloaded MAP is detected according to the load estimation based on handover management cost for each $\mathrm{MN}$ and the handover rate averaged over time of each MN. Then the overloaded MAP initiates the search procedure to find the underutilized MAP and transfer the MN's attachment request to it. Once the attachment transfer is completed, the intended home MAP by COAP and a foreign MAP exchange their roles in the MN attachment phase in a bid to balance handover load.

The remainder of this paper is structured as follows. Section 2 presents the relevant work on load-aware mobility management. The COAP scheme and the key design philosophy of LA-COAP are described in Section 3. Section 4 and 5 portrays the overload detection approach and the attachment transfer scheme respectively. Section 6 discusses the cost of attachment transfer. The numerical results are provided in Section 7. This paper concludes in Section 8.

\section{Related work}

In previous research for MAP selection scheme, many adjusting algorithms and techniques adapted to solve load control problem. Most existing work focuses on load control or control mechanism from the view of MNs. The $\mathrm{MN}$ mobility properties such as velocity and speed are deployed to reduce and relieve MAPs overloaded.

Bandai and Sasase [6] introduced a load balancing mobility management by average BU interval in both AR and MN. When the interval of sending BUs in $\mathrm{MN}$ is shorter than that of receiving BUs in $\mathrm{AR}$, the $\mathrm{MN}$ selects a MAP with largest distance because the MN's movement is estimated to be fast. If the interval of sending BUs in $\mathrm{MN}$ is longer than that of receiving BUS in AR, the MN selects a MAP with the second largest distance. To keep the transparency to HMIPv6, this average BU interval in AR is mapped into the 4 bit binary preference value in the MAP option. Ito and Atsumi [7] proposed a scoring method to select a MAP and to achieve load balancing. The score is calculated from the historical handover frequency and the holding time value. Each MAP holds the management list of MNs sorted by the score. Then the

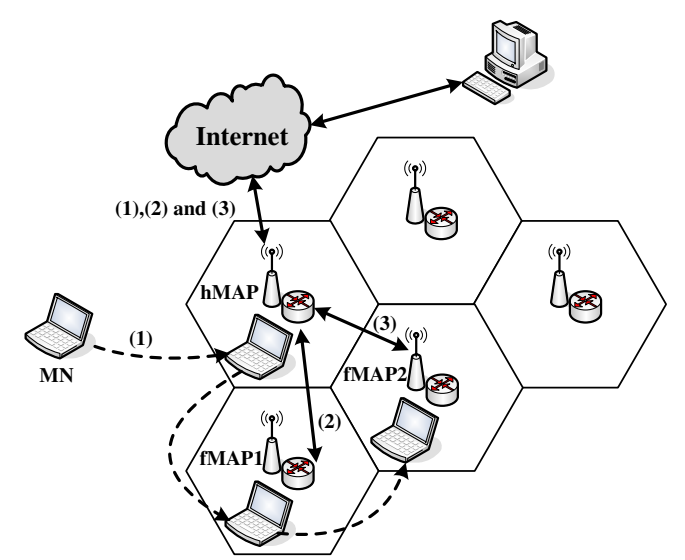

Fig. 1: The movement of MN in 802.11s mesh network.

MAP compares the load with another MAP by requesting the management of MN. The request is to manage MN with the smallest and the highest score on a list until load becomes balanced. In another mechanism, Wang et al. [8] designed the MAP Load Table (MLT) to record the load condition of neighbor MAPs. The scheme will choose the MAP which has minimum load value to register. The mechanism takes the MN's particular characteristics which include the mobility velocity and quantity of communication services. In [9], a dynamic efficient method for selecting the most appropriate MAP is proposed. The operation of the proposed scheme consists of two steps. Initially, the proposed scheme behaves similar to the traditional distance-based MAP selection scheme of HMIPv6. In a given domain, MAPs with loads that are less than a predefined threshold are sorted. The furthest MAP (among these MAPs) is selected first as in the distance-based selection scheme of HMIPv6. When all MAPs have loads that exceed the threshold, the selection of MAPs becomes based on an estimation of MAP load transition using the exponential moving average (EMA) method. Distant MAPs with load decrease tendency are selected first. In [10], a newly defined factor, i.e., the session-to-mobility ratio (SMR), is used as a factor for selecting the serving MAP. SMR is defined as the ratio of the session arrival rate to the handover frequency. In the SMR-based scheme, the highest MAP is selected for MNs with small values of SMR.

\section{COAP scheme}

A network based local mobility scheme based on Cooperative AP (COAP) for 802.11 wireless networks is proposed in [4]. As an alternative to PMIPv6 or network based handover in 802.11 networks, COAP requires neither modification on MN nor APs under the same administrative domain. It use cooperative DHCP server to ensure that wherever $\mathrm{MN}$ roams to in the mesh, it will acquire the same IP address. 
The mobility management for 802.11 based networks under different administrative domains is illustrated in Fig. 1, where each cell represents the coverage of an Access Point (AP) and the corresponding router. In Fig. 1, dashed lines represent the movements of MN, and solid lines represent the forwarding path of inbound and outbound traffic when MN attaches to different MAPs. It is assumed MAPs are under different administrative domains and they collocate with a Mesh Portal (MP) to provide Internet connectivity to mobile users. In Fig. 1, the dashed lines represent the movements of MN. Initially, MN enters the network and attaches to its home MAP hMAP (Step 1), then it moves to a foreign MAP fMAP1 (Step 2). Subsequently, it leaves fMAP1 and enters another foreign MAP fMAP2 (Step 3). Home MAP is always the initial MAP that $\mathrm{MN}$ attaches to and responsible for the mobility management of $\mathrm{MN}$. In addition, hMAP will also take care of the redirection of MN's traffic within the mesh network until MN leaves the network. Each time the handover takes place for MN, hMAP should update its information about MN to track mobility. The traffic caching and forwarding performed by hMAP connecting the Internet ensure that the communication between $\mathrm{MN}$ and $\mathrm{CN}$ is not interrupted by the handover and roaming. We can see that hMAP crowded by $\mathrm{MN}$ is likely to be overloaded by the mobility management cost. In the case of MNs arriving at the network at the unevenly geographically distributed rate, some MAP may be overloaded while some may be light-loaded. This calls for the perception of hMAP's load as well as the load-aware attachment balancing among MAPs.

\section{Overload detection}

The mobility management cost of hMAP for its MNs is determined jointly by the handover management cost for each $\mathrm{MN}$, the handover rate averaged over time of each $\mathrm{MN}$ and the number of MNs registering it as their home MAP.

\subsection{Traffic cache cost of hMAP}

When MN roams to fMAP2 from fMAP1, fMAP1 will send a Client Detached (CD) message to hMAP to notify the movement of $\mathrm{MN}$. Upon receiving the $\mathrm{CD}$ message, the hMAP starts caching the inbound traffic destined to MN. The caching lasts until a Client Attached (CA) message from the fMAP2 arrives at the hMAP. When hMAP receives the notification from the fMAP1, it will release the cached packets to the network.

Since there is no traffic handover between fMAP1 and fMAP2, the CD message should be released in a conservatively early manner. This prevents the traffic loss in the handover between two successive fMAPs because a late CD message may make the traffic destined for IMAP1 from hMAP find the MN already detached. Let $H_{B}^{A}$ represent the hop distance between two nodes A and $\mathrm{B}$. If the expected per hop delay is $\tau$, we can write is the time for fMAP1 to send a CD message to hMAP as

$$
T_{C D}=H_{h M A P}^{f M A P 1}
$$

To avoid traffic loss, fMAP1 has to send a CD message at least $T_{C D}$ before the MN detaches from it. hMAP starts caching the traffic headed for fMAP1 when CD message arrives.

The time for fMAP2 to send a CA message to hMAP can be expressed as

$$
T_{C A}=H_{h M A P}^{f M A P 2}
$$

Let the time taken to perform layer 2 association be $T_{L 2}$ and the time taken to acquire IP address through DHCP be $T_{D H C P}$. The caching duration of hMAP, $T_{C A C H E}$, can be computed as

$$
T_{C A C H E} \geq T_{C D}+T_{L 2}+T_{D H C P}+T_{C A}
$$

Let the time between the delivery of $\mathrm{CD}$ message and MN's detaching is $T_{C D}+\delta, \delta \geq 0$. Then we have

$$
T_{C A C H E}=T_{C D}+\delta+T_{L 2}+T_{D H C P}+T_{C A}
$$

If the average traffic rate between $\mathrm{CN}$ and $\mathrm{MN}$ is $r$, the minimum cache space required for hMAP's mobility management is

$$
S_{C A C H E}=T_{C A C H E} r
$$

Note that $T_{C A C H E}$ is bounded by a timer maintained by hMAP to keep track leased addresses. The timer is designed to identify the departure of $\mathrm{MN}$ from the network and reclaim the address previously leased to $\mathrm{MN}$. The timer is started by the receipt of CD message when hMAP detects MN has detached from fMAP1 and ended by the receipt of $C D$ message when hMAP detects $M N$ has attached to fMAP2. If no CD message is received within MaxHandoverDelay seconds, hMAP assumes the MN has left the network and broadcast a Client Deregistered to all MAPs. The mobility management is completed for the MN by that time. The constraint can be expressed as

$$
\delta+T_{L 2}+T_{D H C P}+T_{C A} \leq \text { MaxHandoverDelay }
$$

So $T_{C A C H E}$ is bounded by (7).

$$
T_{\text {CACHE }} \leq T_{C D}+\text { MaxHandoverDelay }
$$

\subsection{Handover registration cost of hMAP}

To keep track of node mobility, each MAP will maintain a Registered Client Table, which records information about 


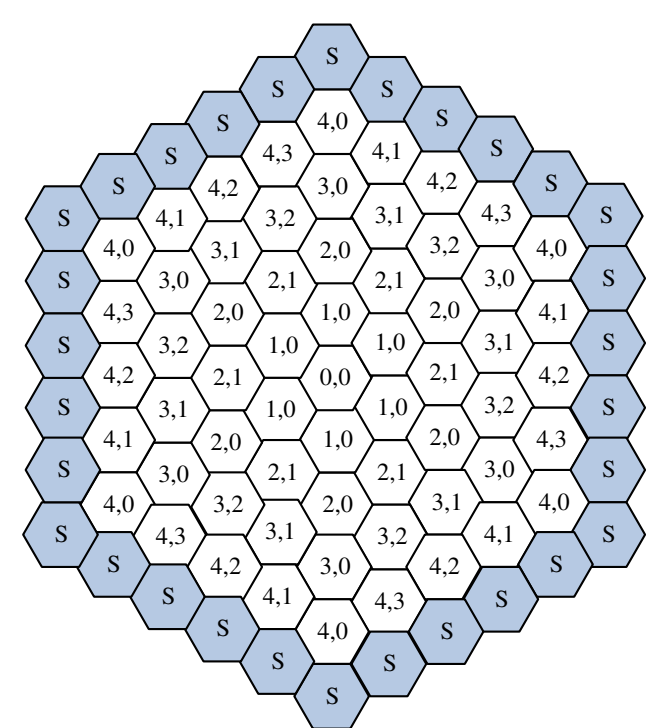

Fig. 2: Cell classification in 5-subarea mesh network.

the nodes who uses this MAP as home MAP. Each entry in Registered Client Table includes the MAC address of $\mathrm{MN}$, the IP address of MN and the MAP MN currently attaches to. The Registered Client Table is used to track the address usage and reclaim address leased when MN leaves the network.

When MN roams to a foreign MAP, fMAP1, fMAP1will notify hMAP, the Home MAP of MN, by sending a Client Attached (CA) message. hMAP will then update its Registered Client Table accordingly. When MN later leaves the coverage of fMAP1, fMAP1 will also send a Client Detached (CD) message to hMAP to notify the movement of $\mathrm{MN}$. Upon receiving the $\mathrm{CD}$ message, hMAP will start a timer to measure the handover delay until the upper limit, MaxHandoverDelay or the receipt of CA message from the next MAP, fMAP2. Let the cost of one time updating of Registered Client Table be $A_{r}$ and the cost of one handover of timer counting be $A_{t}$, then the overall handover registration cost of hMAP can be expressed as

$$
A_{R E G}=A_{r}+A_{t}
$$

\subsection{Handover rate of $M N$}

We assume that each MN follows a random walk model, which ensures that each MN has chances of walking out of the network after enough movements. The movements of $\mathrm{MN}$ within the network coverage result in the crossMAP handovers. Therefore, the number of handovers is dependent of the initial position of $\mathrm{MN}$ in the network.

Inspired by [11], we model the movement of MN as 2D random walk in a hexagonal cluster consisting of hexagonal cells as illustrated in Fig. 2, where each

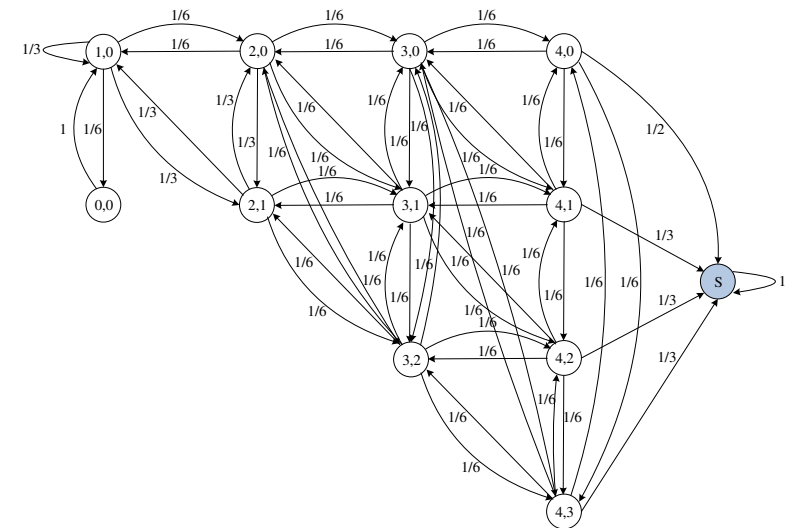

Fig. 3: A state transition diagram illustrating the transition probability between states.

hexagon represents the coverage of a MAP. In Fig. 2, cells are labeled $(x, y)$ where $x$ being the hop count from the center cell and $y$ being the type of the cell. Each unshaded cell represents the coverage of a MAP in the mesh network. The surrounding shaded cells, referred to as boundary cells, are outside of the cluster and beyond the coverage of the mesh network. The movements of each $\mathrm{MN}$ in the mesh network follow a random walk. Whenever an MN moves out of the region, i.e., moving to a boundary cell, the random walk enters an absorbing state. Each cell in the mesh is marked as $(x, y)$ where $x$ represents the hop count distance from the center cell and $y \geq 0$ represents the $y+1$ st type of the cell. Note that there are cells with identical $(x, y)$ label. These cells are considered equivalent because they have the same set of neighbors. For example, cells labeled with $(3,1)$ has common neighbors of $(3,0),(3,2),(2,0),(2,1),(4$, 1) and $(4,2)$.

Therefore the total number of cells can be simplified and reduced. Refer to [11] for the algorithm to label the cells.

Since with random walk, a MN can move to any of the neighboring cells with equal probability, i.e. 1/6 in the case of hexagon, the state transition diagram of the random walk over the reduced set of cells can be derived as illustrated in Fig. 3. A state $(x, y)$ represents $\mathrm{MN}$ in a cell of type $(x, y)$. Because we only interest in the movements of MN within the mesh network, we considers the movement ends once MN leaves the networks, i.e. reaching one of the $\mathrm{S}$ cells. Correspondingly, state $\mathrm{S}$ is an absorbing state, it indicates that MN has leaved the network.

Based on Fig. 3, the transition matrix $\mathbf{P}$ of the random walk can be written as (9). The order of elements in the rows and columns of the matrix follows the order of $(0,0)$, $(1,0),(2,0),(2,1), \ldots,(4,2),(4,3)$ and $S$. Each element $p_{i j}$ of $\mathbf{P}$ represents the probability that $\mathrm{MN}$ will move to state $j$ in the next transition when currently in state $i$. Based 
on Chapman-Kolmogorov equation, the k-step transition matrix $\mathbf{P}^{(k)}$ can be written as (10).

$$
\begin{aligned}
& \mathbf{P}=\left[\begin{array}{ccccccccc}
0 & 1 & 0 & 0 & 0 & \cdots & 0 & 0 & 0 \\
\frac{1}{6} & \frac{1}{3} & \frac{1}{6} & \frac{1}{3} & 0 & \cdots & 0 & 0 & 0 \\
0 & \frac{1}{6} & 0 & \frac{1}{3} & \frac{1}{6} & \cdots & 0 & 0 & 0 \\
0 & \frac{1}{3} & \frac{1}{3} & 0 & 0 & \cdots & 0 & 0 & 0 \\
\vdots & \vdots & \vdots & \vdots & \vdots & \ddots & \vdots & \vdots & \vdots \\
0 & 0 & 0 & 0 & 0 & \cdots & 0 & \frac{1}{6} & \frac{1}{3} \\
0 & 0 & 0 & 0 & 0 & \cdots & \frac{1}{6} & 0 & \frac{1}{3} \\
0 & 0 & 0 & 0 & 0 & \cdots & 0 & 0 & 1
\end{array}\right] \\
& \\
& \mathbf{P}^{(k)}= \mathbf{P}^{k}
\end{aligned}
$$

Each element $p_{i j}^{(k)}$ in $\mathbf{P}^{(k)}$ is the probability of $\mathrm{MN}$ moves from state $i$ to state $j$ in $k$ transitions. Then we can define the probability that MN moves state $j$ from state $i$ at $k$ th transition, $p_{k, i j}$, as follow

$$
p_{k, i j}=\left\{\begin{array}{cc}
p_{i j} & \text { if }(k=1) \\
p_{i j}^{(k)}-p_{i j}^{(k-1)} & \text { if }(k>1)
\end{array}\right.
$$

Besides the number of handovers, the handover rate is also dependent of the handover speed, or the cross-MAP speed.

Since faster-moving MN usually incurs more frequent handovers, we write the handover rate of $\mathrm{MN}$ as

$$
R_{H A N D O V E R}=a * \frac{s_{i}}{C}
$$

Where $s_{i}$ is the moving speed of $\mathrm{MN} i, C$ is the side length of hexagon and $a$ is a constant.

If the number of transitions made by a MN starting in state $\mathrm{i}$ before leaving is $k, k \geq 1$, the residential time can be written as $\frac{k}{R_{\text {HANDOVER }}}$. Then the expected residential time $w_{i}$ of a MN starting in state $i$ before leaving can be computed as

$$
w_{i}=\sum_{k=1}^{\infty} p_{k, i S} * \frac{k}{R_{\text {HANDOVER }}}
$$

The handover rate averaged over time $t, Q_{i}(t)$, for a MN starting in state $i$ is

$$
\begin{array}{r}
Q_{i}(t)=\sum_{k=1}^{\left\lfloor t * R_{\text {HANDOVER }}\right\rfloor} p_{k, i S} * \frac{k}{t}+ \\
\sum_{k=\left\lfloor t * R_{\text {HANDOVER }}\right\rfloor+1}^{\infty} p_{k, i S} * \frac{\left\lfloor t * R_{\text {HANDOVER }}\right\rfloor}{t}
\end{array}
$$

Where $\lfloor *\rfloor$ is the floor function.

\subsection{Overload detection}

The handover management cost for $\mathrm{MN}$ consists of handover registration cost and traffic cache cost of its hMAP.

Based on analysis above, we can conclude the handover management cost for $\mathrm{MN}, A_{H A N D O V E R}$, as

$$
A_{H A N D O V E R}=\omega_{1} A_{R E G}+\omega_{2} T_{C A C H E}
$$

Where $\omega_{1}$ and $\omega_{2}\left(\omega_{1}+\omega_{2}=1,0 \leq \omega_{1}, \omega_{2} \leq 1\right.$, $\omega_{1}=0.55, \omega_{2}=0.45$ in our implementation) are the weights assigned to the handover registration cost and the traffic cache cost.

The handover cost of hMAP per time unit averaged over time $t$ for a MN starting in state $i$ can be written as

$$
U_{i}(t)=Q_{i}(t) * A_{H A N D O V E R}
$$

The expected number of transitions $H_{i}$ made by a MN starting in state $i$ before leaving can be computed as

$$
H_{i}=\sum_{k=1}^{\infty} p_{k, i S} * k
$$

Upon the attachment to a MAP, MN will initiate the DHCP procedure to acquire an IP address from the MAP. When the MAP receives the DHCP request from MN, it will first look up its Mesh Client Table and determine whether this $\mathrm{MN}$ is new to the network or is an existing roaming $\mathrm{MN}$ from other cells. If the $\mathrm{MN}$ is found to be new to the network, the MAP will compute its expected handover cost per time unit averaged over $t$. Each entry in Registered Client Table is calculated by the state of the MAP the MN currently attaches to. Let the number of MAP in state $i$ in Registered Client Table be $N(i)$, $N(i)=0,1,2, \ldots$. The expected handover cost per time unit averaged over $t$ is

$$
\Omega_{i}=\sum_{i=1}^{n(n+1) / 2} U_{i}(t) * N(i)
$$

Here we set a global unique value for $t$, the average number of transitions $H(n)$ made by a $\mathrm{MN}$ in n-subarea mesh network before leaving, which can be computed as

$$
H(n)=\sum_{i=1}^{n(n+1) / 2} H_{i} * \frac{2}{n(n+1)}
$$

When $\Omega_{t}$ is obtained, it is compared with the capability threshold of the MAP. If $\Omega_{t}$ exceeds the capability threshold, the MAP is already overloaded by its attached MNs and has to transfer the current MN's attachment to its neighboring MAPs. If $\Omega_{t}$ falls below the capability threshold, the attachment procedure proceeds and the current MAP will manage the MN's mobility until the MN leaves the network. 


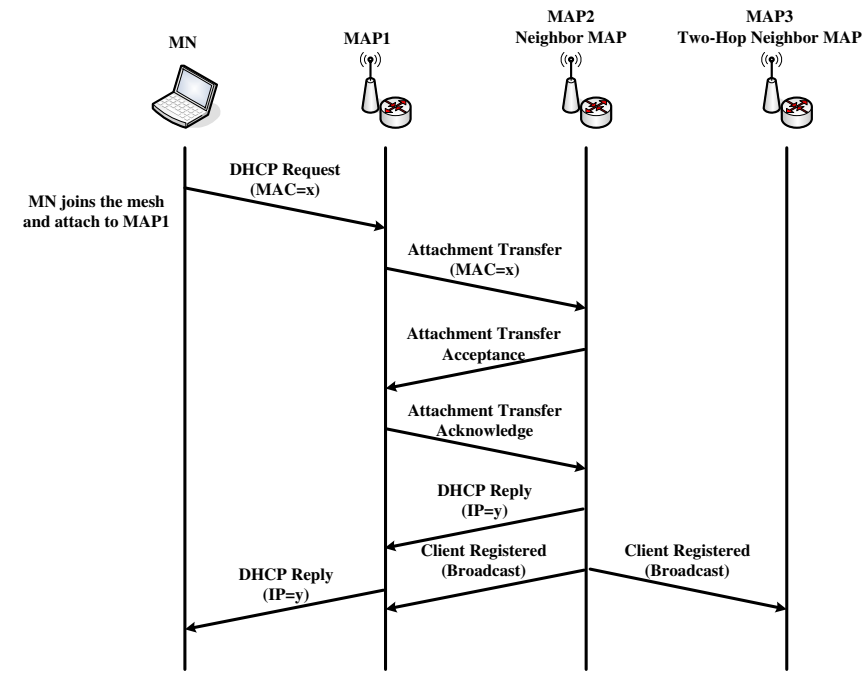

Fig. 4: The signaling of the attachment transfer (ATA from neighbor).

\section{Attachment transfer}

The main idea of the attachment transfer is that the overloaded MAP may transfer the MN's attachment request to the light-loaded MAP, therefore foreign MAP and home MAP exchange their roles in the MN attachment phase in a bid to balance handover load.

For the attachment transfer, the current MAP will send Attachment Transfer (AT) message to all its neighbors containing the MAC address of MN and the MAP MN currently attaches to. Upon the receipt of AT message, each neighbor will compute the expected handover cost per time unit averaged over $t$ and determine whether it has the capability to accept AT accordingly. If a neighbor is available for AT, it will notify the current MAP attached by MN with Attachment Transfer Acceptance (ATA) message. Otherwise, the response is Attachment Transfer Denied (ATD) message. If more than one neighbor responses with ATA message, the current MAP can choose from the responding MAPs according to some optimization criteria. e.g. for the sake of load balance, the least attached home MAP found in the Mesh Client Table among all responding MAPs may be preferable as the AT destination. Then the current MAP sends Attachment Transfer Acknowledge (ATAck) message to the AT destination MAP. When receiving ATAck message, the AT destination MAP will assign an IP address to MN from its address pool. The MAP MN currently attaches to successfully transfers its MN to the AT destination MAP and becomes the foreign MAP. At the same time, the AT destination MAP becomes the MN's home MAP. Afterwards, the hMAP will update its Registered Client Table with the IP address of MN, the MAC address of MN and the MAP MN currently attaches to ( both in the previous AT message ). hMAP will also broadcast a Client Registered message to other MAPs,

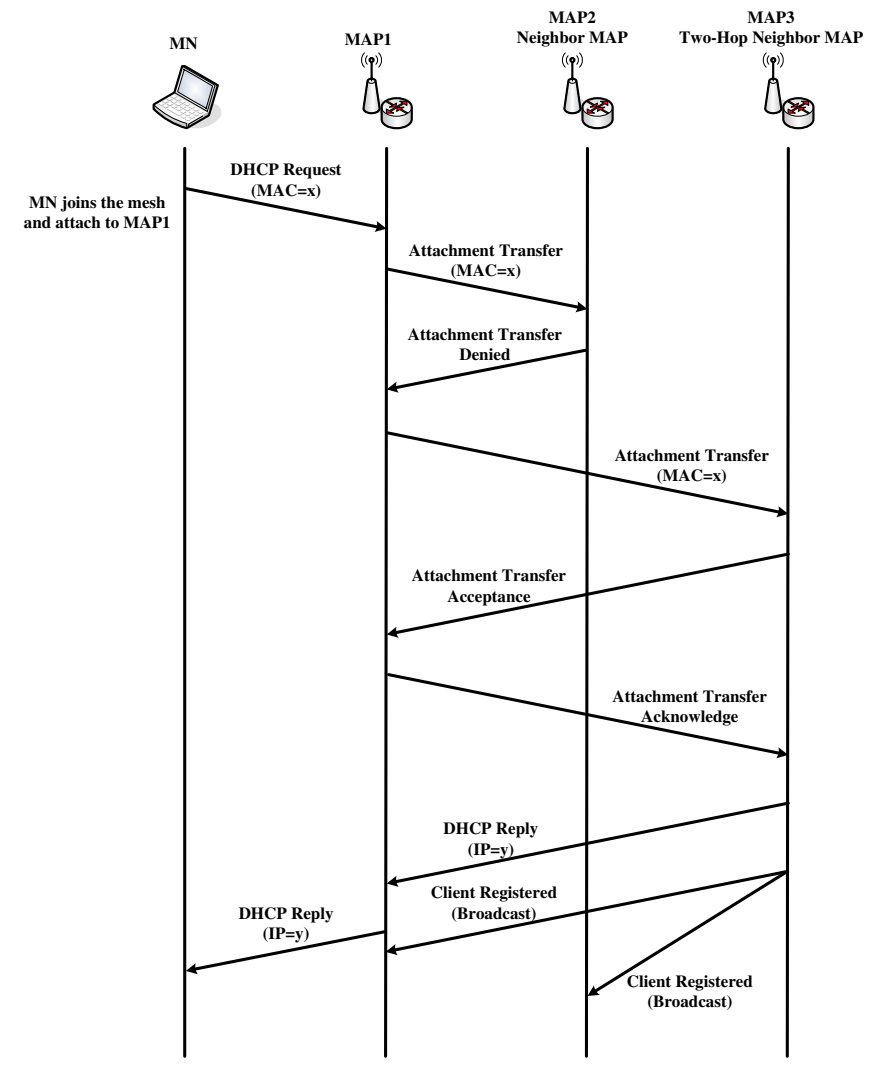

Fig. 5: The signaling of the attachment transfer (ATA from twohop neighbor).

indicating the MAC address of MN, IP address assigned and the corresponding Home MAP. Other MAPs will record this information in their Mesh Client Table together with the IP address of the announcing MAP. The signaling of the attachment transfer is illustrated in Fig. 4 (ATA from neighbor).

If all neighbors response with ATD message, the current MAP will search two-hop neighbors for the potential AT destination MAP. The procedure is carried out similar to the two-hop neighbor AT. The three-hop neighbor search is initiated if the two-hop search fails, and so on. In the worst case, the AT attempt fails if all other MAPs in the network deny the AT. The signaling of the attachment transfer is illustrated in Fig. 5 (ATA from two-hop neighbor).

In order to identify the distances between itself and the other MAPs,each MAP will broadcast Neighbor Distance Discovery (NDD) messages to its neighbors during startup of the network. Each neighbor returns a Neighbor Distance Discovery Acknowledge (NDDA) message to the source MAP of the NDD message notifying the hop distance between them. Each neighbor also forwards the NDD message to all its neighbors except the incoming MAP of the NDD message. Then every MAP receiving the NDD message also notifies the source MAP of the hop distance between them. The NDD 
dispersion procedure lasts for enough time until no more NDDA message is replied to the source MAP. The NDD scheme ensures that each MAP has knowledge about the distances to the other MAPs, which facilitates the aforementioned step wise search approach of the potential AT destination MAP.

Note that the AT destination MAP search approach puts priority on the nearest neighbor available, thereby minimizes the attachment transfer delay. Section 6 will provide further numerical analysis of the attachment transfer delay. Another advantage of this step wise search approach lies in the diminished number of AT messages compared with the simple broadcast of AT messages for the whole network. However, sometimes it is necessary to tradeoff between the search delay and the number of AT messages for the search. e.g. simultaneous search of the one-hop and two-hop neighbors makes it possible to speed the search procedure meanwhile the number of AT messages is unlikely to rise sharply.

\section{Cost analysis of attachment transfer}

To compute the delay of attachment transfer, let's consider the process when MN attempts to attach to MAP1 and MAP1 is detected as overloaded. The number of i-hop $(i=1,2, \ldots, n)$ neighbor of MAP1 is $m_{i}$. Each MAP in the network except MAP1 has an overload probability, which is denoted by $e$. The AT search follows one-hop wise search approach. We define the delay of attachment transfer as the period from the time $\mathrm{MN}$ joins the mesh and sends DHCP request to MAP1 until it attaches to MAP1 and starts receiving the data traffic from $\mathrm{CN}$.

We can derive the expected delay of attachment transfer, $T_{L A-C O A P}^{\text {Attach }}$, as

$E\left(T_{L A-C O A P}^{A t t a c h}\right)=\sum_{i=1}^{n} T_{i}^{A T}\left(1-e^{m_{i}}\right) \Pi_{j=1}^{i-1} e^{m_{j}}+T_{n}^{A T F} \Pi_{i=1}^{n} e^{m_{i}}$

Where $T_{i}^{A T}$ represents the delay of attachment transfer via i-hop $(i=1,2, \ldots, n)$ neighbor of MAP1 and $T_{n}^{A T F}$ represents the delay of failure of attachment transfer via overload detection (no MAP is available).

To compute $T_{i}^{A T}$, we can see that the overload detection of previous j-hop $(j=1,2, \ldots, i-1)$ neighbor of MAP1 takes the delay of $2 j \tau$ (Attachment Transfer + Attachment Transfer Denied) . But the i-hop neighbor

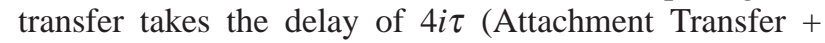
Attachment Transfer Acceptance + Attachment Transfer Acknowledge + DHCP Reply). Therefore, we can write $T_{i}^{A T}$ as

$$
T_{i}^{A T}=T_{L 2}+2 T_{D H C P}+4 i \tau+\sum_{j=1}^{i-1} 2 j \tau
$$

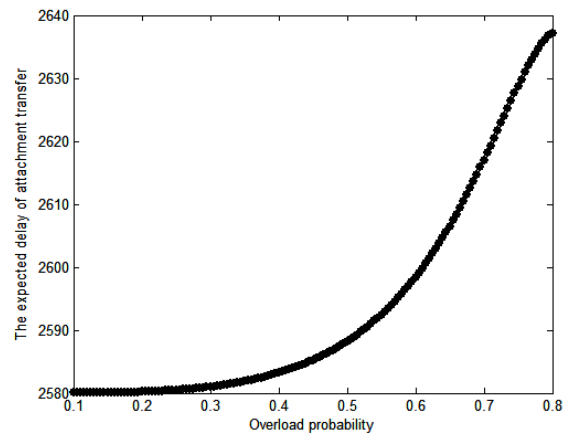

Fig. 6: The expected delay of attachment transfer of LA-COAP for different overload probability $(n=4)$.

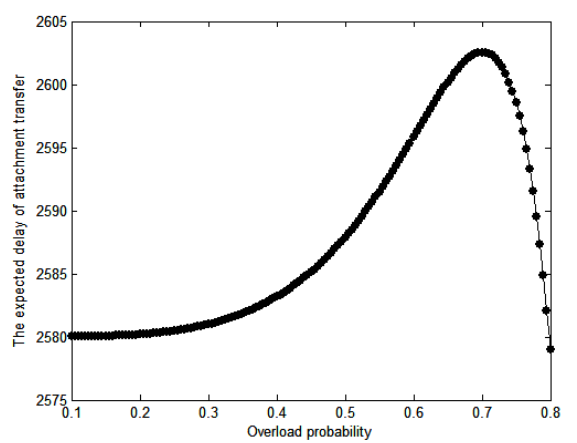

Fig. 7: The expected delay of attachment transfer of LA-COAP for different overload probability $(n=3)$.

Similarly, we can derive $T_{n}^{A T F}$ as (22) noting that i-hop $(i=1,2, \ldots, n)$ neighbor of MAP1 all takes the delay of $2 i \tau$ (Attachment Transfer + Attachment Transfer Denied) .

$$
T_{n}^{A T F}=T_{L 2}+T_{D H C P}+\sum_{i=1}^{n} 2 i \tau
$$

In comparison, the expected delay of $\mathrm{MN}$ attachment of the original load-oblivious COAP, $T_{C O A P}^{A t t a c h}$, is

$$
T_{C O A P}^{\text {Attach }}=T_{L 2}+2 T_{D H C P}
$$

We can also write the expected number of messages for the attachment transfer, $U_{L A-C O A P}^{\text {Attach }}$, as

$$
E\left(U_{L A-C O A P}^{A t t a c h}\right)=\sum_{i=1}^{n}(2 i+2)\left(1-e^{m_{i}}\right) \Pi_{j=1}^{i-1} e^{m_{i}}+2 n \Pi_{i=1}^{n} e^{m_{i}}
$$

\section{Numerical results}

Let $T_{L 2}=500 \mathrm{~ms}, T_{D H C P}=1000 \mathrm{~ms}, \tau=20 \mathrm{~ms}, m_{i}=4(i=$ $1,2, \ldots, n)$. 


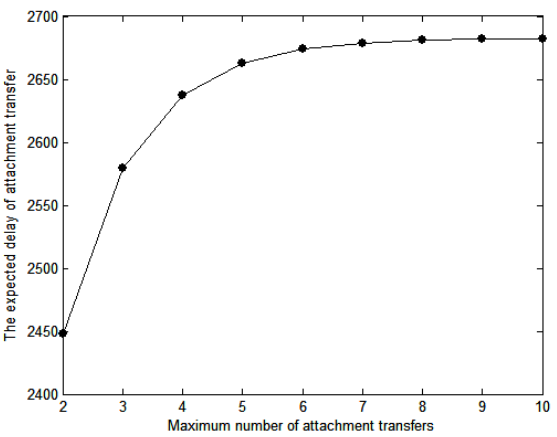

Fig. 8: The expected delay of attachment transfer of LA-COAP for different maximum number of attachment transfers $(e=0.8)$.

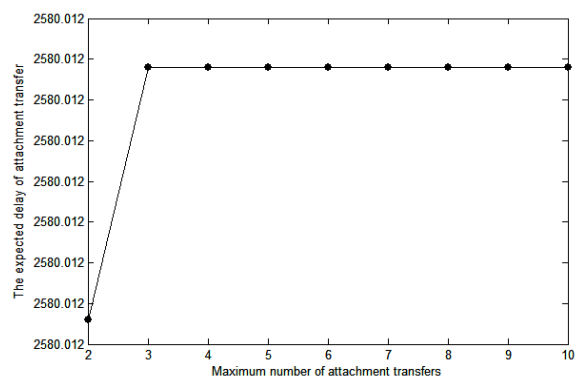

Fig. 9: The expected delay of attachment transfer of LA-COAP for different maximum number of attachment transfers $(e=0.1)$.

Let $n=4$, the expected delay of attachment transfer of LA-COAP for different overload probability can be plotted in Fig. 6. It can be seen that as overload probability increases, the handover delay of LA-COAP increases accordingly. High overload probability means LA-COAP tends to make more AT searches for attachment transfer, therefore takes more time. Let $n=3$, the numerical results are shown in Fig 7. In comparison with Fig. 6, the handover delay decreases for larger overload probability. This is due to the fact that for small $n$ the probability that LA-COAP ends with failure of attachment transfer is high. And if $T_{n}^{A T F}$ takes less time than $T_{i}^{A F}$, the handover may incur less delay instead for larger overload probability .

Let $e=0.8$, the expected delay of attachment transfer of LA-COAP for different maximum number of attachment transfers can be plotted in Fig. 8. The handover delay increases as the maximum number of attachment transfers increases as illustrated in Fig. 8. However, for $e=0.1$, we can see in Fig. 9 that the difference of handover delay for varied maximum number of attachment transfers is minor.

To plot the numerical result of $U_{L A-C O A P}^{\text {Attach }}$, we similarly use $n=4$ for different overload probability (as shown in Fig. 10) and for different maximum number of

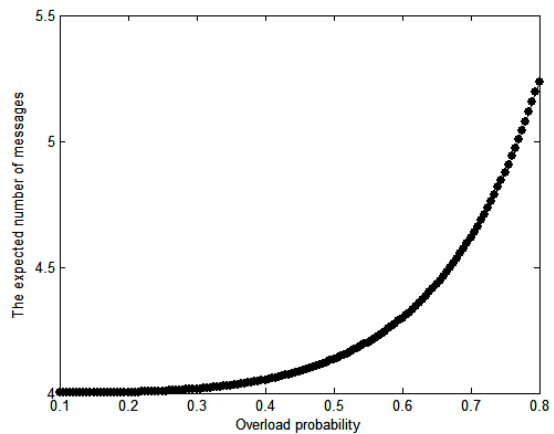

Fig. 10: The expected number of messages of LA-COAP for different overload probability.

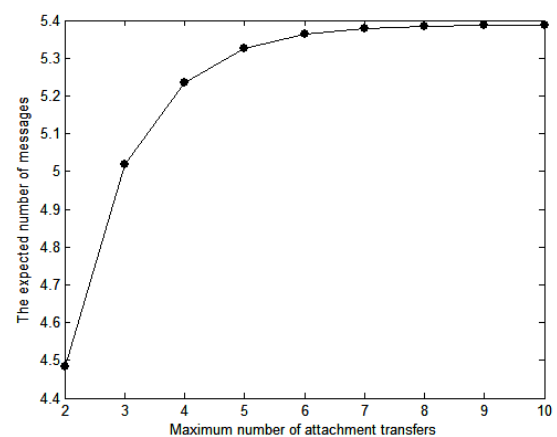

Fig. 11: The expected number of messages of LA-COAP for different maximum number of attachment transfers.

attachment transfers (as shown in Fig. 11) respectively. The results suggests that the increase of overload probability and maximum number of attachment transfers both incur larger $U_{L A-C O A P}^{\text {Attach }}$.

\section{Conclusion}

To overcome the load-oblivious problem involved in the COAP scheme, this paper proposes a load-aware mobility management scheme. The proposed scheme can efficiently balance the handover load among MAPs at the cost of slightly prolonged attachment delay and increased attachment messages compared with COAP.

\section{Acknowledgement}

This work was supported in part by the National Key Technology R\&D Program of China under the grant number 2012BAH16B00 and the National Science Foundation of China under the grant number 61003239. 


\section{References}

[1] G. Hiertz, D. Denteneer, S. Max, R. Taori, J. Cardona, L. Berlemann, and B. Walke, IEEE Wireless Communications, 17, 104-111 (2010).

[2] L. H. Yen, T. T. Yeh, and K.H. Chi, IEEE Internet Computing, 13, 30-38 (2009).

[3] S. G. Ed., K. Leung, V. Devarapalli, K. Chowdhury, and B. Patil, Internet Request for Comments, RFC 5213, (2008).

[4] Y. Xia, C. K. Yeo, Proceedings of the IEEE 76th Vehicular Technology Conference, (2012).

[5] H. Soliman, C. Catelluccia, K. El Malki, and L. Bellier, Internet Request for Comments, RFC 4140, (2005).

[6] M. Bandai and I. Sasase, Proceedings of the IEEE PIMRC, 2003, 460-464 (2003).

[7] K. Ito and A. Atsumi, Proceedings of the 6th AsiaPacific Symposium on Information and Telecommunication Technologies, (2006).

[8] Y. H. Wang, K. F. Huang, C. S. Kuo, and W.J. Huang, Proceedings of the 22nd International Conference on Advanced Information Networking and Applications, 691696 (2008).

[9] T. Taleb, A. Jamalipou, Y. Nemoto and N. Kato, IEEE Transactions on Vehicular Technology, 58, 954-964 (2009).

[10] S. Pack, M. Nam, T. Kwon, and Y. Choi, Computer Communications, 29, 3066-3078 (2006).

[11] I. F. Akyildiz, Y. B. Lin, W. R. Lai, and R. J. Chen, IEEE Journal on Selected Areas in Communications, 18, 12541260 (2000).

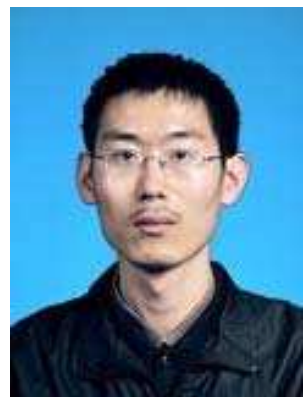

Zheng Wang received the MS degree in Electrical Engineering from Institute of Acoustics, Chinese Academy of Sciences in 2006, and the $\mathrm{PhD}$ degree in Computer Science from Computer Network Information Center, Chinese Academy of Sciences in 2010. He is currently the director of Joint Labs in China Organizational Name Administration Center. His research interests are in the areas of network architecture, Domain Name System, and information systems. 